\title{
EXPLORING THE IMPACT OF ENTERPRISE SOCIAL MEDIA AFFORDANCES ON WORK PERFORMANCE AND KNOWLEDGE TRANSFER
}

\author{
Ravoniarivelo Ravaka Andrianina, Zhejiang Gongshang University, Hangzhou, Zheijang, Peoples Republic of \\ China
}

\author{
dx.doi.org/10.18374/IJBR-21-1.5
}

\begin{abstract}
One of the derivatives of collective work is knowledge, and this remains a significant resource to any organization, either valued as capital or part of the intangible assets of that organization. The effective application of knowledge, through processing and transfer mediums can impact positively work performance. There have been noted studies on enterprise social media affordances with regards to the use of different platforms and the user experiences though these studies did not take into consideration how the design differences impacted the performance of enterprise social media platforms. To fill this knowledge gap, this research focused on determining the impact that a singular enterprise social media platform has on work performance, within the framework of knowledge transfer. This research collected data from 317 employees of a Malagasy company using the internal social media platform and it was found that the affordances of association, persistence, visibility and editability had a positive relationship with knowledge acquisition and knowledge provision, and these in turn also promoted work performance among them. The research also showed that there is a mediation effect of knowledge acquisition on the impact of ESM affordances on work performance. A key finding of this research was also in the fact that through the knowledge transfer process, organizations can co-create value in a sustainable way that impacts performance, and this is aided by the innovation process, along with the levers for action on the variables. The essential variables for which actions need to be taken are derived from the complex relationships of technology (ESM affordances), knowledge transfer (knowledge provision and knowledge acquisition) and organizational performance (work performance and task performance).
\end{abstract}

Keywords: Enterprise Social Media Affordances, Work Performance, Knowledge Transfer 